\title{
Percepção de profissionais de enfermagem sobre lesões por pressão relacionadas a dispositivos médicos
}

\author{
Perception of nursing professionals about evidence by area related to medical devices \\ Percepción de los profesionales de enfermería sobre la evidencia por áreas relacionadas con \\ dispositivos médicos
}

Sabrina Guterres da Silva Galetto ${ }^{1}$ (1) Eliane Regina Pereira do Nascimento ${ }^{2}$ (1) Patricia Madalena Vieira Hermida ${ }^{3}$ (1) Daniele Delacanal Lazzari² Nara Reisdorfer ${ }^{2}$ (1) Josefine Busanello ${ }^{4}$ (1)

1. Hospital Universitário Polydoro Ernani de São Thiago. Florianopolis, SC, Brasil.

2. Universidade Federal de Santa Catarina. Florianópolis, SC, Brasil.

3. Secretaria Municipal De Saúde.

Florianópolis. SC, Brasil.

4. Universidade Federal do Pampa.

Uruguaiana, RS, Brasil.

\section{Resumo}

Objetivo: Conhecer a percepção de profissionais de enfermagem atuantes em unidade de terapia intensiva acerca das lesões por pressão relacionadas a dispositivos médicos. Método: Pesquisa qualitativa, descritiva, realizada com 12 profissionais de enfermagem de um hospital público de ensino de Santa Catarina. A coleta de dados se deu por entrevista semiestruturada, $e$ análise pela técnica do discurso do sujeito coletivo com o emprego do software QualiQuantiSoft $\circledast$. Resultados: Emergiram cinco Ideias Centrais: tipos de dispositivos e ocorrência das lesões por pressão relacionadas a dispositivos médicos; (in)visibilidade e (des)valorização destas lesões no cuidado ao paciente crítico; lesões por pressão relacionadas a dispositivos médicos podem ser inevitáveis; perfil do paciente crítico e risco para desenvolver a lesão; e (des)conhecimento profissional sobre o impacto da lesão na vida das pessoas após alta da terapia intensiva. Conclusão e implicações para a prática: A percepção da enfermagem acerca das lesões por pressão relacionadas a dispositivos médicos está vinculada aos tipos de dispositivos, a ocorrência das lesões na terapia intensiva, ao cuidado ofertado e ao impacto das lesões na vida das pessoas.

Palavras-chave: Enfermagem; Lesão por pressão; Cuidados críticos; Cuidados de enfermagem; Segurança do Paciente.

\section{Abstract}

Objective: To know the perception of Nursing professionals working in intensive care units regarding medical device-related pressure injuries related. Method: A qualitative and descriptive research, carried out with 12 Nursing professionals from a public teaching hospital in Santa Catarina. Data collection took place through semi-structured interviews, and the analysis was performed using the collective subject discourse technique by means of the QualiQuantiSoft $\circledast$ Software. Results: Five Central Ideas emerged: types of devices and the occurrence of medical device-related pressure injuries; (in)visibility and (de)valuation of these injuries in the care of critical patients; medical device-related pressure injuries can be inevitable; critical patient profile and risk to develop the injury; and (lack of) professional knowledge about the impact of the injury on people's lives after discharge from intensive care. Conclusion and implications for the practice: The perception of Nursing about medical device-related pressure injuries is linked to the types of devices, the occurrence of injuries in intensive care, the care offered and the impact of injuries on people's lives.

Keywords: Nursing; Pressure injury; Critical care; Nursing Care; Patient Safety.

\section{REsumen}

Objetivo: Conocer la percepción de los profesionales de Enfermería que trabajan en unidades de cuidados intensivos acerca de las lesiones por presión relacionadas con dispositivos médicos. Método: Investigación cualitativa y descriptiva, realizada con 12 profesionales de Enfermería de un hospital escuela público Santa Catarina. Los datos se recolectaron por medio de una entrevista semiestructurada, y el análisis tuvo lugar a través de la técnica de discurso del sujeto colectivo utilizando el software

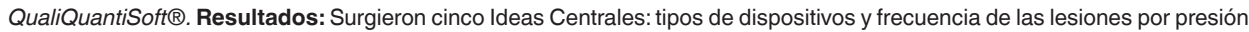
relacionadas con dispositivos médicos; la (in)visibilidade y (des)valorización de estas lesiones en la atención al paciente en estado crítico; las lesiones por presión relacionadas con dispositivos médicos pueden ser inevitables; perfil del paciente en estado crítico y riesgo de desarrollar una lesión; y (des)conocimiento profesional sobre el efecto de la lesión en la vida de las personas después del alta de la unidad de cuidados intensivos. Conclusión e implicaciones para la práctica: La percepción del personal de Enfermería acerca de las lesiones por presión relacionadas con dispositivos médicos está vinculada a los tipos de dispositivos, a la frecuencia de las lesiones en la unidad de cuidados intensivos, a la atención prestada y al efecto de las lesiones en la vida de las personas.

Palabras clave: Enfermería; Lesión por presión; Cuidado crítico; Atención de Enfermería; Seguridad del Paciente.
Autor correspondente:

Sabrina Guterres da Silva Galetto.

E-mail: sabrinaguterres@gmail.com.

Recebido em 07/07/2020.

Aprovado em 12/09/2020.

DOl:https://doi.org/10.1590/2177-9465-EAN-2020-0225
Palabras clave: Entermeria, Lesión por presión; Cuidado crilico; Atención de Entermeria, Seguidad del Paciente. 


\section{INTRODUÇÃO}

A assistência de enfermagem no contexto da terapia intensiva tem evoluído significativamente ao longo dos anos. Esse fato culmina com surgimento de procedimentos cada vez mais complexos e uso de muitos dispositivos médicos, que embora essenciais no cuidado aos pacientes críticos, podem causar eventos adversos, a exemplo das lesões por pressão relacionadas a dispositivos médicos (LP RDM). ${ }^{1}$

Resultado do uso de dispositivos criados e aplicados para fins diagnósticos e terapêuticos, as LP RDM geralmente apresentam o padrão ou forma do dispositivo e devem ser categorizadas de acordo com o sistema de classificação de lesões por pressão. ${ }^{2}$ Essas lesões podem se desenvolver em qualquer parte do corpo em que os dispositivos médicos estejam inseridos. Os locais comumente relatados incluem cabeça, face, pescoço e extremidades, associados principalmente a dispositivos de imobilização como talas, colares cervicais; e dispositivos respiratórios como tubos endotraqueais, traqueostomia e máscaras de ventilação não invasiva. ${ }^{3}$

Pacientes dependentes de dispositivos médicos, como aqueles que estão gravemente doentes, têm maior chance de desenvolver lesões por pressão. ${ }^{4}$ Estudos revelam que as taxas de LP RDM podem variar de 1,7\% a $86 \%$ em pacientes de unidades médico-cirúrgicas e de Unidades de Terapia Intensiva (UTI), respectivamente, e geralmente não são identificadas até atingirem estágios 3 ou $4 .^{5}$

A equipe de enfermagem exerce papel fundamental na identificação e prestação de cuidados para prevenir e/ou minimizar a ocorrência das LP RDM. Contudo, esses profissionais nem sempre conhecem os riscos dos dispositivos médicos no desenvolvimento de lesões. Nesse sentido, pesquisa realizada na Turquia evidenciou que $20 \%$ dos enfermeiros não acreditavam que os dispositivos médicos pudessem causar lesão por pressão. ${ }^{6}$ Ademais, alguns profissionais não reconhecem as LP RDM como lesões por pressão, seja porque desconhecem a terminologia do National Pressure Injury Advisory Panel (NPIAP) ou por discordarem dela. ${ }^{7}$

Isso mostra a necessidade de ampliar as discussões acerca das LP RDM, que embora tenham ocorrência significativa, nem sempre recebem a devida atenção da equipe de saúde, o que justifica a realização desta pesquisa, também sustentada por uma revisão integrativa ${ }^{8}$ que recomenda novos estudos sobre o tema nos diferentes cenários do cuidado, em especial no Brasil, devido às diversas realidades assistenciais. À vista disso, esta investigação objetivou conhecer a percepção de profissionais de enfermagem atuantes em unidade de terapia intensiva acerca das lesões por pressão relacionadas a dispositivos médicos.

Destaca-se que esta pesquisa pode conferir visibilidade às LP RDM no contexto de cuidados críticos e contribuir com o planejamento de ações para prevenir a ocorrência das mesmas.

\section{MÉTODO}

Estudo descritivo, qualitativo, desenvolvido na UTI geral de um hospital público de ensino do estado de Santa Catarina. No período da coleta de dados, a UTI contava com 10 leitos ativos para internação de pacientes clínicos agudizados e cirúrgicos (cirurgia geral, do aparelho digestivo, vascular, oncológicas, do aparelho urinário e ginecológicas). A estrutura física dispunha de dois postos de enfermagem, rouparia, expurgo, sala de equipamentos, almoxarifado, sala de limpeza e copa. A área administrativa possui sala para chefias médica, de enfermagem e de fisioterapia, biblioteca, descansos médico e de enfermagem, salas para visitantes, reuniões e aulas.

O quadro de profissionais de enfermagem era composto por: 1 chefia de enfermagem, 14 enfermeiros assistenciais e 38 técnicos de enfermagem. Participaram os profissionais da equipe de enfermagem que atenderam aos critérios de inclusão: ser enfermeiro ou técnico de enfermagem da UTI há pelo menos seis meses e estar em exercício profissional. Foram excluídos profissionais em períodos de férias ou licenças de qualquer natureza. Empregou-se a amostragem intencional.

Os dados foram coletados no período de fevereiro a abril de 2018, por meio de entrevista semiestruturada, realizada por uma das pesquisadoras. Na sala de reunião da UTI, as entrevistas foram individuais, a partir da questão norteadora: Como você percebe a problemática das lesões por pressão relacionadas a dispositivos médicos na unidade de terapia intensiva? As entrevistas tiveram duração média de 20 minutos, foram gravadas e posteriormente transcritas na íntegra. A amostragem foi por saturação teórica, ${ }^{9}$ conforme os passos: 1. Disponibilizar os registros de dados "brutos" - à medida que as entrevistas eram realizadas se procedia à sua transcrição; 2. "Imergir" em cada registro - concomitante ao passo anterior, fez-se a leitura das transcrições para identificar seus núcleos de sentido; 3. Compilar as análises individuais - realizou-sea compilação de temas e/ou enunciados identificados nos depoimentos; 4. Reunir os temas e/ou enunciados - falas com mesmos núcleos de sentido foram agrupadas; 5. Codificar ou nominar os dados simultaneamente ao passo anterior foi realizada a nominação dos enunciados; 6 . Alocar os temas e/ou enunciados-em uma tabela, alocou-se os enunciados destacando quando se deu a primeira ocorrência; 7. Constatar a saturação teórica - a visualização da saturação teórica ocorreu quando após novas entrevistas não foram acrescentados enunciados diferentes. ${ }^{9}$ Assim, identificou-se que após a sexta entrevista não houve novos enunciados, porém, seguiu-se com a coleta de dados até a décima segunda entrevista, no intuito de reforçar a saturação.

Para organização dos dados, utilizou-se o software QualiQuantiSoft $囚$ versão 1.3.c., e para a análise, a técnica do Discurso do Sujeito Coletivo ${ }^{10}$ (DSC) que dispõe de quatro figuras metodológicas: expressões chaves $(\mathrm{ECH})$ - são os extratos mais significativos dos depoimentos; ideias centrais (IC) - nome ou expressão linguística que descreve o sentido de cada conjunto homogêneo de $\mathrm{ECH}$; ancoragem (AC) compreende pressupostos, princípios, hipóteses e teorias que dão sustentação às expressões chave; e DSC - é um discurso síntese redigido na primeira pessoa do singular, com $\mathrm{ECH}$ que têm IC ou AC semelhantes ou complementares. ${ }^{10}$

O software QualiQuantiSoft $\circledast$ compreende quatro componentes: 1) Cadastros: nesse campo foram cadastradas todas as informações individuais dos participantes (ex: idade, sexo, formação, etc..), além das perguntas utilizadas na entrevista e as respostas transcritas de cada entrevistado; 2) Análises: esse campo é chamado de Instrumento de Análise do Discurso (IAD), o qual é dividido em 
IAD1 e IAD2. No IAD1 foi realizada a análise de cada entrevista, selecionando-se os trechos mais significados $(E C H)$, e em seguida, identificando-se as IC. O IAD2 auxiliou na construção do DSC a partir do agrupamento de todas as ECH com a mesma IC; 3) Ferramentas: permitiu exportar os resultados da pesquisa do software para o Microsoft巴 Office Word versão 2010;4) Relatórios: possibilitou gerar relatórios dos DSC, síntese das IC e percentual de participação de cada entrevistado nos DSC.

Destaca-se que o uso de software de análise para o tratamento dos dados, permite maior credibilidade, confirmabilidade, coerência e confiabilidade na pesquisa qualitativa, diminuindo os vieses dos pesquisadores. ${ }^{11}$ Ainda, para assegurar a confirmabilidade e credibilidade do estudo, o material empírico oriundo da coleta de dados foi revisado por pares, ${ }^{12}$ incluindo a pesquisadora que coletou os dados e outras duas doutoras em enfermagem envolvidas no estudo, com embasamento teórico-metodológico correspondente. Ressalta-se a relevância da validação dos pesquisadores no exercício da pesquisa por estar associada aos critérios de confirmabilidade e credibilidade. ${ }^{11}$

Os participantes identificados nos discursos pelas letras "E" de entrevistado, atribuindo-se números de acordo com a sequência das entrevistas (E1, E2, E3...). A pesquisa seguiu as orientações e disposições da Resolução no 466/12 e foi aprovada pelo Comitê de Ética em Pesquisa com Seres Humanos da Universidade Federal de Santa Catarina Parecer № 2.091404 em 30 de maio de 2017. Todos participantes assinaram o Termo de Consentimento Livre e Esclarecido.

\section{RESULTADOS}

Participaram do estudo 12 profissionais de enfermagem, dos quais sete técnicos e cinco enfermeiros, a maioria $(n=10)$ do sexo feminino. A idade variou entre 21 e 51 anos (média 40 anos). O tempo de formação referente ao cargo compreendeu de quatro a 25 anos (média de 16 anos) e o tempo médio de atuação em terapia intensiva foi de 9,6 anos.

Quanto à titulação acadêmica, dos técnicos de enfermagem, seis possuíam ensino superior completo, quatro em enfermagem e dois em outras áreas de conhecimento, além de especializações, sendo três em terapia intensiva e dois em outras áreas. Os cinco enfermeiros entrevistados possuem mestrado com dissertação na área de terapia intensiva, um é doutor em enfermagem e um estava cursando doutorado em enfermagem.

A análise dos depoimentos originou cinco IC com seus respectivos DSC sobre as LP RDM. A figura metodológica AC não foi identificada nos depoimentos dos profissionais.

A IC1 "Tipos de dispositivo e ocorrência das LP RDM na UTI" emergiu do depoimento de cinco profissionais, enquanto as IC2 e IC3, respectivamente, "Perfil do paciente crítico e risco para desenvolver LP RDM" e "(In)visibilidade e (des)valorização das LP RDM no cuidado ao paciente crítico", representam os depoimentos de seis participantes. Outras IC foram originadas das falas de um número menor de profissionais: IC4 "LP RDM podem ser inevitáveis" e IC5 "(Des)conhecimento do profissional sobre o impacto das LP RDM na vida das pessoas após alta da UTI", que correspondem ao discurso de dois e um participante, respectivamente.

\section{IC1:Tipos de dispositivo e ocorrência das LP RDM na UTI}

DSC1: Eu acho que por ser UTI, pelo fato de ter vários dispositivos invasivos a questão se torna mais prevalente. Principalmente pacientes graves, de isolamento. As lesões por pressão pelos dispositivos são causadas pela intubação, pela sondagem, cateter venoso central ou periférico. Enfim, tudo aquilo que se usa como terapia no paciente e que tem como secundário essa lesão. Eu acho que todo dia conseguimos ver um caso, às vezes até mais de uma lesão no mesmo paciente. Ultimamente tenho visto com mais frequência ou não observava tanto. Não sei se realmente houve um aumento dessas lesões por dispositivos ou se as pessoas começaram a me chamar a atenção para isso. Notamos, infelizmente, só na hora do banho onde tem a lesão e o que está causando. Porque é quando se troca o cadarço, se tira a monitorização do paciente pra ver, é onde se olha com mais critério para o paciente. Acho que todo cuidado de higiene e conforto está relacionado com observar esse tipo de coisa, as fixações, os drenos, as sondas, se não estão machucando a pele. (E1, E3, E6, E9, E10)

\section{IC2: Perfil do paciente crítico e risco para desenvolver LP RDM}

DSC2: São vários fatores que contribuem para a lesão como o dispositivo, o tempo, a gravidade. Normalmente está relacionada também com droga vasoativa, que faz uma fragilidade, uma predisposição. Alguns pacientes têm mais propensão, aqueles com uma pele mais macia, com mais edema, pacientes mais idosos, com uma pele mais friável, acabam lesionando. Os que são mais agitados a fixação do tubo tem que ficar mais forte, já costumam fazer lesão de orelha. (E1, E2, E5, E6, E8, E12)

\section{IC3: (In)visibilidade e (des)valorização das LP RDM no cuidado ao paciente crítico}

DSC3: Tenho observado que muitas vezes acabamos não dando tamanha importância as lesões por dispositivos quando comparadas às lesões que envolvam proeminências ósseas, por exemplo. Muitas vezes isso passa despercebido. Essas lesões têm um valor muito pequeno diante de um número tão grande de cuidados que prestamos, diante das coisas que temos que observar. Mas achei bem interessante essa questão vir à tona, é uma coisa nova. Na verdade, ouvi falar na faculdade e no curso técnico, mas aqui na UTI não falamos tanto nisso. Nós falamos muito em lesão por pressão tradicional, de região sacra, mas somos confrontados muitas vezes com esse tipo de 
lesão por dispositivos. Realmente é algo que devemos ter um pouco mais de atenção. (E3, E4, E5, E7, E9, E11)

\section{IC4: LP RDM podem ser inevitáveis}

DSC4: Tem casos que se entende que é muito difícil evitá-las [as lesões] em função da necessidade do dispositivo, que por ser um corpo estranho vai gerar um efeito deletério. Porque tem todo um contexto de condição do paciente, como uso de droga vasoativa, obesidade, enfim, que é inerente ao paciente não relacionado aos cuidados que implementamos. Porque por mais que tu cuides, tem paciente que não tem como evitar. (E4, E5)

\section{IC5: Desconhecimento profissional sobre o impacto das LP RDM na vida das pessoas após alta da UTI}

DSC5: Não consigo avaliar com que grau de debilidade o paciente sai da UTI. Porque muitos dos pacientes que recebem alta médica saem com alguma lesão daqui, e não consigo acompanhar que tipo de efeito deletério surgiu ou surge na vida do paciente nas atividades de vida diária fora daqui. Um exemplo: um paciente masculino que sai com uma lesão de meato uretral pela sonda vesical, que chega a quase fissurar o pênis... não tenho a informação de como é que ele lida com esse sofrimento, com esse dano, com essa lesão lá na frente. Se ele passa por algum tipo de cirurgia plástica, se ele faz algum tratamento específico. Como que o paciente e família lidam com isso, não temos conhecimento. Onosso cuidado ainda fica muito limitado à Unidade de Terapia Intensiva, porque há lesões "simples" como uma lesão de asa de nariz por sonda nasoenteral como uma lesão mais "grave" como essa de meato uretral que vai requerer um tratamento médico que pode limitar a pessoa em algumas atividades específicas, algumas atividades de vida diária. (E4)

As percepções dos profissionais de enfermagem acerca das LP RDM, expressas nos discursos, foram sintetizadas e são apresentadas no Quadro 1.

Quadro 1. Síntese das percepções dos profissionais acerca das lesões por pressão relacionadas a dispositivos médicos. Florianópolis, Santa Catarina, Brasil, 2018.

\begin{tabular}{|c|c|c|}
\hline Ideias Centrais & DSC & Percepções dos profissionais \\
\hline \multirow{4}{*}{$\begin{array}{l}\text { Tipos de dispositivo e ocorrência das LP } \\
\text { RDM na UTI }\end{array}$} & \multirow{4}{*}{ DSC1 } & $\begin{array}{l}\text { - Acreditam que pacientes internados em UTI estão mais suscetíveis à } \\
\text { LP RDM. }\end{array}$ \\
\hline & & $\begin{array}{l}\text { - Definem LP RDM como dano secundário a um procedimento } \\
\text { terapêutico ao paciente crítico (ex: intubação, sondagem). }\end{array}$ \\
\hline & & $\begin{array}{l}\text { - Percebem diariamente casos de LP RDM, inclusive mais de uma no } \\
\text { mesmo paciente. }\end{array}$ \\
\hline & & - Utilizam o banho de leito para identificação das LP RDM. \\
\hline $\begin{array}{l}\text { Perfil do paciente crítico e risco para } \\
\text { desenvolver LP RDM }\end{array}$ & DSC2 & $\begin{array}{l}\text { - Citam como fatores de risco o uso de dispositivos, tempo de } \\
\text { internação, a gravidade, isolamento de contato, uso de droga } \\
\text { vasoativa, edema, idade avançada, agitação psicomotora. }\end{array}$ \\
\hline \multirow{2}{*}{$\begin{array}{l}\text { (In)visibilidade e (des)valorização das LP } \\
\text { RDM no cuidado ao paciente crítico }\end{array}$} & \multirow{2}{*}{ DSC3 } & $\begin{array}{l}\text { - Costumam prestar mais atenção nas lesões por pressão tradicionais } \\
\text { que acometem proeminências ósseas. }\end{array}$ \\
\hline & & $\begin{array}{l}\text { - Reconhecem uma (des)valorização das LP RDM no contexto de } \\
\text { cuidados críticos. }\end{array}$ \\
\hline \multirow[b]{2}{*}{ LP RDM podem ser inevitáveis } & \multirow[b]{2}{*}{ DSC4 } & $\begin{array}{l}\text { - Entendem que em alguns casos, mesmo com cuidados, as LP RDM } \\
\text { são inevitáveis. }\end{array}$ \\
\hline & & $\begin{array}{l}\text { - Citam a necessidade permanente do dispositivo, condição clínica e } \\
\text { características inerentes ao paciente como fatores que interferem na } \\
\text { prevenção. }\end{array}$ \\
\hline \multirow{2}{*}{$\begin{array}{l}\text { (Des)conhecimento profissional sobre o } \\
\text { impacto das LP RDM na vida das pessoas } \\
\text { após alta da UTI }\end{array}$} & \multirow[b]{2}{*}{ DSC5 } & $\begin{array}{l}\text { - Referem dificuldade em mensurar o impacto que as LP RDM causam } \\
\text { na vida dos pacientes após a alta. }\end{array}$ \\
\hline & & $\begin{array}{l}\text { - Refletem sobre o sofrimento dos pacientes e de seus familiares e } \\
\text { acerca da possível necessidade de tratamentos adicionais para reparar } \\
\text { o dano causado pelas LP RDM. }\end{array}$ \\
\hline
\end{tabular}




\section{DISCUSSÃO}

O DSC 1 evidencia que a percepção dos profissionais sobre o tema estudado está centrada nos tipos de dispositivo e ocorrência das LP RDM no contexto da terapia intensiva. Foram abordadas definições e etiologia das LPRDM, que convergem com a definição da National Pressure Injury Advisory Panel, quando refere que as lesões por pressão relacionadas a dispositivos são resultado do uso de dispositivos criados e aplicados para fins diagnósticos e terapêuticos. ${ }^{2}$ A compreensão desse fenômeno é primordial na medida em que se pensa em prevenção, tendo em vista que a falta de conscientização e reconhecimento das lesões por pressão pela equipe, são fatores de riscos adicionais para seu desenvolvimento.,

Os profissionais relataram observar diariamente casos de LP RDM, inclusive mais de uma no mesmo paciente. Estudo realizado em uma UTI da Arábia Saudita, mostrou que 26,7\% dos pacientes tiveram pelo menos uma LP RDM e 10,4\% desenvolveram mais de uma lesão. ${ }^{13}$

Ainda no DSC1, a identificação dos casos de LP RDM foi mencionada pelos participantes. Sabidamente, na assistência de enfermagem o banho é um cuidado no qual se realiza inspeção cutânea criteriosa, visando sua integridade. Quando se trata de LP RDM, inspeção da pele é premissa importante para promover o cuidado centrado e de qualidade. ${ }^{14}$ Para além desta avaliação, é essencial que enfermeiros priorizem a identificação precoce destas lesões, por meio de inspeções regulares da pele, minimamente uma vez a cada turno, particularmente no caso de pacientes com fatores de risco para o desenvolvimento de lesões, tais como edema localizado ou sistêmico. ${ }^{6,15}$

A compreensão dos profissionais sobre o risco para o desenvolvimento de LP RDM, presente no DSC2, está em consonância com a literatura. Estudo australiano identificou que pessoas internadas em UTI estão 3,8 vezes mais propensas a desenvolver uma lesão por pressão quando comparadas às internadas nas enfermarias. Entre os fatores que justificam essa vulnerabilidade, estão a própria doença crítica, instabilidade hemodinâmica, imobilidade prolongada e uso de múltiplos dispositivos médicos. ${ }^{4}$

Nesse discurso, os profissionais ainda expressaram que pacientes com agitação psicomotora são mais vulneráveis à ocorrência de LP RDM, percepção que encontra respaldo em um estudo multicêntrico que avaliou os efeitos da não sedação sobre a ocorrência de lesões por pressão em pacientes sob ventilação mecânica. Os resultados revelaram que pacientes não sedados apresentaram maior prevalência de LP RDM quando comparados aos que foram sedados. ${ }^{16}$ Em contrapartida, a sedação pode acarretar maior tempo de internação em UTI, comprometendo a mobilidade física, aumentando o tempo de ventilação mecânica e contribuindo para necessidade de uso prolongado de dispositivos médicos. ${ }^{5}$

Embora os profissionais percebam que são recorrentes as LP RDM na UTI (DSC1), reconhecem uma (des)valorização dessas no contexto de cuidados críticos, como mostra o DSC3. No discurso dos participantes é atribuído "um valor muito pequeno" a essas lesões diante da dimensão do cuidado prestado em terapia intensiva. Essa questão foi também observada em pesquisa realizada por enfermeiras australianas, na qual os profissionais declararam que quando as prioridades vitais entram em cena, problemas como as LP RDM são negligenciados. ${ }^{5}$

Essa "(in)visibilidade" das LP RDM foi chamada de "Epidemia Oculta", no sentido de que historicamente esse tipo de lesão não tem recebido a devida atenção. ${ }^{17}$ Fatores como elevada carga de trabalho, quantitativo de pessoal inadequado e escassez de recursos e treinamento, podem ter relação direta com o cuidado dispendido às lesões por pressão, sendo esses elementos apontados por profissionais de enfermagem como as principais barreiras para prevenção dessas lesões. ${ }^{18}$ Apesar da evidenciada (in) visibilidade das LP RDM no DSC3, os profissionais reconhecem a necessidade de dar maior atenção a esses eventos adversos.

No DSC4, os participantes expressaram que em alguns casos, mesmo que cuidados preventivos sejam realizados, as lesões tendem a ocorrer. As LP RDM são mais complicadas de prevenir quando comparadas às lesões por pressão tradicionais, pois os dispositivos podem ser um componente diagnóstico/ terapêutico essencial no tratamento, e nem sempre seu reposicionamento é possível. ${ }^{5}$ Além disso, a condição clínica dos pacientes como anemia, baixos níveis de albumina, hipotensão, terapia vasopressora e ventilação mecânica, podem elevar o risco das LP RDM. ${ }^{7}$

Uma das dificuldades mencionadas pelos enfermeiros no DSC5, é a de mensurar o impacto que as LP RDM causam na vida dos pacientes após sua alta. Foi citado exemplo de lesão por pressão no meato uretral pela sonda vesical de demora (SVD) e, refletiu-se brevemente sobre o sofrimento implicado a esses indivíduos e seus familiares e acerca da possível necessidade de tratamentos adicionais para reparar esse dano. Especificamente nos casos de lesões por pressão causadas por sonda vesical, estudo realizado em Israel, evidenciou uma prevalência de $36 \%$ de LP em meato uretral de homens pelo uso de SVD. ${ }^{19}$ Nesses casos pode ser necessária a reconstrução uretral e/ou desvio urinário, com implicações físicas e psicológicas para a pessoa pela dor e desfiguração da imagem corporal. ${ }^{20}$

Obviamente, é necessário considerar repercussões a posteriori, contudo, é imprescindível também compreender os efeitos desses agravos ainda no contexto de cuidados críticos, ponderando que LP RDM podem contribuir para processos infecciosos e dor, impactando inclusive no aumento do tempo de internação em UTI.

Diante do exposto, ressalta-se a necessidade de maior vigilância e conscientização da equipe de enfermagem e multiprofissional para os casos de LP RDM, pois resultados satisfatórios são alcançados quando todos os membros da equipe compreendem e valorizam seu papel na prevenção desse agravo ${ }^{7}$ prevenção essa que requer intervenções baseadas em evidências e divulgação das informações para toda a equipe multiprofissional. ${ }^{14}$ 
Por reconhecer que a identificação e prevenção das LP RDM é responsabilidade de toda equipe multiprofissional, uma limitação do estudo foi a abordagem restrita aos profissionais de enfermagem. Aponta-se também como limitação, o fato das entrevistas não terem sido submetidas à validação pelos participantes, o que associada a revisão por pares realizada, poderia conferir maior credibilidade ao estudo. ${ }^{12}$

\section{CONSIDERAÇÕES FINAIS E IMPLICAÇÕES PARA A PRÁTICA}

A percepção dos profissionais de enfermagem acerca das lesões por pressão relacionadas a dispositivos médicos compreende o reconhecimento dos dispositivos que causam as lesões, bem como a ocorrência destas e sua identificação diária na UTI, principalmente durante o banho realizado pela enfermagem. Os participantes acreditam que pacientes em UTI estão mais suscetíveis a essas lesões e reconhecem uma (in)visibilidade e (des)valorização desses eventos adversos no contexto de cuidados críticos. Para os profissionais, em alguns casos as LP RDM são inevitáveis e quando ocorrem não conseguem mensurar o seu impacto na vida das pessoas após alta da UTI.

Acredita-se que esta pesquisa despertou reflexões individuais e coletivas na equipe de enfermagem sobre a problemática das LP RDM no cenário da terapia intensiva, pois colocou em pauta um tema pouco explorado no cotidiano do trabalho, conforme exposto pelos profissionais, de modo a sensibilizar a mesma quanto à necessidade de vigilância e cuidados para prevenir ou minimizar a ocorrência desses eventos adversos. Cabe ainda, recomendar novos estudos que envolvam também outros profissionais de saúde.

\section{CONTRIBUIÇÕES DOS AUTORES}

Desenho do estudo. Sabrina Guterres da Silva Galetto. Eliane Regina Pereira do Nascimento

Coleta ou produção dos dados. Sabrina Guterres da Silva Galetto

Análise de dados e interpretação dos resultados. Sabrina Guterres da Silva Galetto. Eliane Regina Pereira do Nascimento. Patricia Madalena Vieira Hermida. Danielle Delacanal Lazzari. Josefine Busanello. Nara Reisdorfer

Redação e revisão crítica do manuscrito. Sabrina Guterres da Silva Galetto. Eliane Regina Pereira do Nascimento. Patricia Madalena Vieira Hermida. Danielle Delacanal Lazzari. Josefine Busanello. Nara Reisdorfer

Aprovação da versão final do artigo. Sabrina Guterres da Silva Galetto. Eliane Regina Pereira do Nascimento. Patricia Madalena Vieira Hermida. Danielle Delacanal Lazzari. Josefine Busanello. Nara Reisdorfer

Responsabilidade por todos os aspectos do conteúdo e a integridade do artigo publicado. Sabrina Guterres da Silva Galetto. Eliane Regina Pereira do Nascimento. Patricia Madalena
Vieira Hermida. Danielle Delacanal Lazzari. Josefine Busanello. Nara Reisdorfer

\section{EDITOR ASSOCIADO}

\author{
Cristina Rosa Baixinho
}

\section{REFERÊNCIAS}

1. Hampson J, Green C, Stewart J, Armitstead L, Degan G, Aubrey A et al. Impact of the introduction of an endotracheal tube attachment device on the incidence and severity of oral pressure injuries in the intensive care unit: a retrospective observational study. BMC Nurs. 2018;17(4):1-8 http://dx.doi.org/10.1186/s12912-018-0274-2. PMid:29449786.

2. Edsberg LE, Black JM, Goldberg M, McNichol L, Moore L, Sieggreen M. Revised national pressure ulcer advisory panel pressure injury staging system: revised pressure injury staging system. J Wound Ostomy Continence Nurs. 2016;43(6):585-97. http://dx.doi.org/10.1097/ WON.0000000000000281. PMid:27749790.

3. Ham WH, Schoonhoven L, Schuurmans MJ, Leenen LPH. Pressure ulcers in trauma patients with suspected spine injury: a prospective cohort study with emphasis on device-related pressure ulcers. Int Wound J. 2017;14(1):104-11. http://dx.doi.org/10.1111/iwj.12568. PMid:26767917.

4. Coyer F, Miles S, Gosley S, Fulbrook S, Sketcher-Baker K, Cook JL et al. Pressure injury prevalence in intensive care versus non-intensive care patients: a state-wide comparison. Aust Crit Care. 2017;30(5):244-50. http://dx.doi.org/10.1016/j.aucc.2016.12.003. PMid:28063724.

5. Barakat-Johnson M, Barnett C, Wand T, White K. Medical device-related pressure injuries: an exploratory descriptive study in an acute tertiary hospital in Australia. JTissue Viability. 2017;26(4):246-53. http://dx.doi. org/10.1016/j.jtv.2017.09.008. PMid:29050901.

6. Karadag A, Hanönü SC, Eyikara EA. Prospective, descriptive study to assess nursing staff perceptions of and interventions to prevent medical device-related pressure injury. Ostomy Wound Manage. 2017;63(10):3441. PMid:29091036.

7. Delmore B, Ayello EA. Pressure injuries caused by medical devices and other objects: a clinical update. Am J Nurs. 2017;117(12):36-45 PMid:29120893.

8. Galetto SGS, Nascimento ERP, Hermida PMV, Malfussi LBH. Medica device-related pressure injuries: an integrative literature review. Rev Bras Enferm. 2019;72(2):505-12. http://dx.doi.org/10.1590/0034-71672018-0530. PMid:31017216.

9. Fontanella BJB, Luchesi BM, Saidel MGB, Ricas J, Turato ER, Melo DG Amostragem em pesquisas qualitativas: proposta de procedimentos para constatar saturação teórica. Cad Saude Publica. 2011;27(2):389-94 http://dx.doi.org/10.1590/S0102-311X2011000200020. PMid:21359475.

10. Lefevre F, Lefevre AMC. Pesquisa de Representação Social: um enfoque qualiquantitativo a metodologia do discurso do sujeito coletivo. 2. ed. Brasília: Liber Livro; 2012.

11. Souza No RA, Dias GF, Silva RR, Ramos ASM. Efeitos dos softwares de análise de dados qualitativos na qualidade de pesquisas. Rev. Adm. Contemp. 2019;23(3):373-94. http://dx.doi.org/10.1590/19827849rac2019170357.

12. Moreira $\mathrm{H}$. Critérios e estratégias para garantir o rigor na pesquisa qualitativa. R Bras Ens Ci Tecnol. 2018;11(1):405-24. http://dx.doi. org/10.3895/rbect.v11n1.6977.

13. Faisal AM, Rasheed AM, Parameaswari PJ, Numan OS, Muteb M. A cross-sectional study on medical device-related pressure injuries among critically ill patients in Riyadh, Kingdom of Saudi Arabia. WCET Journal [Internet]. 2017; [citado 2020 jun 6];37(1):8-11. Disponível em: https://search. informit.com.au/documentSummary;dn=705144608520918;res=IELHEA

14. Cavalcanti EC, Kamada I. Medical-device-related pressure injury on adults: an integrative review. Texto Contexto Enferm. 2020;29:e20180371. http://dx.doi.org/10.1590/1980-265x-tce-2018-0371.

15. Kim JY, Lee YJ. Medical device-related pressure ulcer (MDRPU) in acute care hospitals and its perceived importance and prevention 
performance by clinical nurses. Int Wound J. 2019;16(Supl. 1):51-61. http://dx.doi.org/10.1111/iwj.13023. PMid:30793861.

16. Nedergaard HK, Haberlandt T, Toft $P$, Jensen HI. Pressure ulcers in critically ill patients-Preventable by non-sedation? A substudy of the NONSEDA-trial. Intensive Crit Care Nurs. 2018;44:31-5. http://dx.doi. org/10.1016/j.iccn.2017.09.005. PMid:28988757.

17. Baharestani M. Medical device related pressure ulcers: the hidden epidemic across the life span [Internet]. Westford: National Pressure Ulcer Advisory Panel; 2013 [citado 2020 jun 6]. Disponível em: http:// www.npuap.org/wpcontent/uploads/2012/01/7-Final-Baharestani -Medical-Device-RelatedPressure-Ulcers-1The-Hidden-EpidemicAcross-the-Lifespan.pptx.pdf
18. Mirshekari L, Tirgari B, Forouzi MA. Intensive care unit nurses' perceived barriers towards pressure ulcer prevention in south east Iran. J Wound Care. 2017;26(3):145-51. http://dx.doi.org/10.12968/jowc.2017.26.3.145. PMid:28277998.

19. Shenhar C, Mansvetov M, Baniel J, Golan S, Aharony S. Catheterassociated meatal pressure injury in hospitalized males. Neurourol Urodyn. 2020;39(5):1456-63. http://dx.doi.org/10.1002/nau.24372. PMid:32339318.

20. Bell MA. Severe indwelling urinary catheter-associated urethral erosion in four elderly men. Ostomy Wound Manage. 2010;56(12):36-9. PMid:21205992. 